\title{
Use of Sensory Methods for Detecting Target Engagement in Clinical Trials of New Analgesics
}

\author{
Boris A. Chizh* and Christine N. Sang ${ }^{\dagger}$ \\ *GlaxoSmithKline, Addenbrooke's Centre for Clinical Investigation, Addenbrooke's Hospital, Cambridge CB2 2 GG, United \\ Kingdom; and Department of Anesthesiology, Perioperative and Pain Medicine, Brigham and Women's Hospital, Harvard \\ Medical School, 75 Francis Street, Boston, Massachusetts 02115
}

\begin{abstract}
Summary: The translation of analgesic efficacy seen in preclinical pain models into the clinic is problematic and is associated with a number of factors that may result in the failure of clinical trials to detect the effect of investigational therapeutic agents. The use of translational pain biomarkers in phase I trials can potentially reduce some of these risks by measuring the interaction between the drug and its target (termed target engagement) in humans. To serve this purpose, sensory tests and other measures of pharmacological activity in nociceptive pathways need to be identified,
\end{abstract}

based on the preclinical profile of the drug being tested and the feasibility of human assessments. Here we discuss some examples to assess the utility of sensory and related pain biomarkers in the early phase of evaluation of novel analgesics for confirmation of target engagement in humans. The emphasis is on the TRPV1 antagonists, but some other target mechanisms are also discussed in examining the validity of this approach. Key Words: Analgesics, clinical trials, biomarkers, sensory testing, capsaicin, heat, TRPV1, hyperalgesia.

\section{INTRODUCTION}

The transition between preclinical and clinical development phases is probably the highest hurdle for any drug, particularly for a novel analgesic. The large number of uncertainties, any of which could cause failure to translate preclinical efficacy into the clinic, includes differences in target pharmacology between preclinical species and humans, differences in the target role in mechanisms of animal and human pain states, differences in pharmacokinetics (PK), and the difficulty in predicting pharmacologically active concentrations in humans, as well as differences in adverse effects and toxicity.

Phase I clinical trials usually serve to address the issues of safety, tolerability, and PK in humans, and standard phase I studies are not usually designed to confirm therapeutic target engagement. Most commonly, efficacy is assessed by taking a safe and well-tolerated dose (sometimes the maximum tolerated dose) into a phase II proof-of-concept study in a target patient pop-

Address correspondence and reprint requests to: Boris A. Chizh, M.D., Ph.D., Platform Director, Pain \& Neurophysiology, GlaxoSmithKline, Addenbrooke's Centre for Clinical Investigation, Addenbrooke's Hospital, Box 128, Hills Road, Cambridge CB2 2GG, UK. E-mail: boris.a.chizh@ gsk.com. ulation. If those findings are positive, the compound then progresses to further development in large-scale phase $\mathrm{IIb} / \mathrm{III}$ studies. More often than not, however, the outcome of proof-of-concept studies is negative; in such cases, many of the questions mentioned above remain unanswered, of which the most important question is whether or not the target has been invalidated so that no further work in this area is warranted.

The need to change this paradigm is illustrated by the average success rate for all therapeutic areas from 1992 to 2000 for 10 large pharmaceutical companies in the United States and Europe. ${ }^{1}$ Only $11 \%$ of all compounds made it through clinical development during those years; the success rate was lower (8\%) in central nervous system disorders, including chronic pain.

Sensory testing paradigms may serve as biomarkers of disease mechanisms, and thus help align preclinical and clinical development phases for new analgesics. These aspects have recently been reviewed in some detail by several authors. ${ }^{2,3}$ Here, we will discuss the use of sensory tests as efficacy biomarkers in early clinical development. We discuss the use of nociceptive pharmacodynamic (PD) markers in phase I-II and analyze several examples of their application in clinical trials, including the application of relevant experimental methods to eval- 
uate target engagement in the context of novel TRPV1 antagonists.

\section{MECHANISM-BASED CLINICAL BIOMARKERS IN PHASE I CLINICAL TRIALS}

Phase I clinical trials, although focused on human safety and PK endpoints, are opportunities for demonstrating PD effects in humans. This would potentially indicate whether there is receptor occupancy resulting in a functional effect, whether the effects are related to the pharmacokinetics of the compound (PK/PD relationships), and whether there is any separation of PD efficacy from the adverse effects and toxicological limits at the doses studied (therapeutic index).

For some targets, chemical biomarkers may be identified and used to estimate the level of target engagement. This is often done ex vivo, as when the blood of a drug-exposed human subject is treated with challenge agents to evoke a pharmacological response (e.g., release of prostanoids in whole blood as a measure of cyclooxygenase activity ${ }^{4}$ ). Although seemingly simple and sufficiently robust (and potentially translatable between species), such markers do not test the target engagement of novel analgesics in the relevant tissue or pathway, and have not yet been validated. To achieve this, and specifically to potentially make accurate dose predictions, specific PD markers are needed that are sensitive to the target activity in nociceptive pathways or pain perception. We will review some of these sensory markers in the sections that follow.

\section{CASE STUDIES WITH TRPV1 ANTAGONISTS}

A large body of evidence has indicated a role for transient receptor potential vanilloid type 1 (TRPV1), a nonselective cation channel protein expressed predominantly by sensory neurons, in the detection of noxious painful stimuli such as capsaicin, acid, and heat. ${ }^{5}$ The behaviors associated with pain evoked by thermal stimuli are attenuated in TRPV1 null $(-/-)$ mice. $^{6}$ Pharmacological developments aimed at this target soon followed these findings. ${ }^{7}$ These included the discovery of relevant compounds and the testing of the lead compounds in animal models, demonstrating their efficacy in inflammatory and neuropathic pain, as has been summarized in several recent reviews. ${ }^{7,8}$

TRPV1 antagonists seem particularly suitable for testing PD effects in phase I, because several types of challenge involving this target can be performed in healthy volunteers. TRPV1 is activated by capsaicin, noxious heat $\left(>42^{\circ} \mathrm{C}\right)$ and protons $(\mathrm{pH}<6)$, and can be sensitized to heat or protons by prostaglandins and several other proinflammatory agents. ${ }^{9-11}$ Thus, attenuation of heat pain, antagonism of capsaicin-evoked pain or flare, and alleviation of inflammatory hyperalgesia can all potentially serve as PD markers of TRPV1 antagonism in humans. To date, only a few such clinical studies have been reported, and only one has been published as a comprehensive article on the PD effects of a TRPV1 antagonist, SB-705498, in healthy humans. ${ }^{12}$ This example will be used to examine the translation of the preclinical profile of TRPV1 antagonists into humans.

\section{EVALUATION OF SB-705498, A NOVEL TRPV1 ANTAGONIST}

SB-705498, $N$-(2-bromophenyl)- $N^{\prime}-[((R)-1$-(5-trifluoromethyl-2-pyridyl)pyrrolidin-3-yl)]urea (SB-705498), is a potent and selective multimodal TRPV1 antagonist. ${ }^{13}$ Whole-cell patch-clamp electrophysiology showed that SB705498 causes rapid and reversible inhibition of the capsaicin-mediated $\left(\mathrm{IC}_{50}=3 \mathrm{nmol} / \mathrm{L}\right)$, acid-mediated $(\mathrm{pH}=$ $5.3)$, or heat-mediated $\left(50^{\circ} \mathrm{C} ; \mathrm{IC}_{50}=6 \mathrm{nmol} / \mathrm{L}\right)$ activation of human TRPV1 (at $-70 \mathrm{mV}) .{ }^{13}$ In vivo, the compound was shown to significantly attenuate inflammatory hyperalgesia evoked by intraplantar Freund's complete adjuvant in guinea pigs. ${ }^{14}$ Therefore, the following experimental paradigms were considered to assess target engagement in phase I clinical trials and used to evaluate SB-705498 in human volunteers ${ }^{12}$ : the capsaicin challenge, heat pain measurements, and assessment of inflammatory hyperalgesia induced by UV irradiation.

\section{EXPERIMENTAL PAIN MODELS}

\section{Use of intradermal or topical capsaicin}

Either of two formulations of capsaicin may be used in this model. Intradermal (ID) capsaicin formulation is prepared from purified capsaicin powder and dissolved in Tween-80 in water at a concentration of $10 \mathrm{mg} / \mathrm{mL}$. Such injections cause pain, flare and hypersensitivity to heat (heat hyperalgesia at the site of injection), pin-prick and light touch (mechanical hyperalgesia and allodynia in the area extending beyond the injected site). ${ }^{15-18} \mathrm{Si}$ mone et al. ${ }^{17}$ showed that injection of this vehicle alone does not cause secondary hyperalgesia; Sang et al. ${ }^{18}$ showed that the spread of capsaicin cannot account for extension of hyperalgesia into adjacent nerve territories. Capsaicin cream (Axsain, 0.075\% capsaicin w/w) may be applied to the skin on the volar aspect of one arm, left on the skin under occlusion for a period of time (e.g., 30 $\mathrm{min}$ ) and then gently wiped off. This method of capsaicin administration also evokes pain, flare and hyperalgesia (as assessed in pilot experiments, data not shown). The topical formulation of capsaicin was used in the data presented below, primarily because of its ease of administration and relatively low intensity of evoked pain ( $v s$ 
ID injections) which was unlikely to interfere with other assessments.

\section{UVB model}

UV irradiation of the skin in humans is an established experimental method to evoke controlled skin inflammation and inflammatory hyperalgesia. ${ }^{19-21}$ To account for differences in individual sensitivity to UV, the minimum erythema dose for irradiation for each subject is established using a calibrated UV source (B spectrum, 290$320 \mathrm{~nm}$ ). This is done by irradiating several spots with increasing intensities of UVB light. Approximately $24 \mathrm{~h}$ later, threshold erythema is assessed either visually or using colorimetry. The dose of irradiation in the model is $3 \times$ the minimum erythema dose, and subjects who do not develop threshold erythema at $1 / 3$ of the maximum dose that can be safely administered to the subjects are excluded from the clinical trial.

Assessments of skin blood flow and heat pain threshold or tolerance are performed before and immediately after capsaicin application, and also before and $24 \mathrm{~h}$ after the UVB irradiation. Heat pain may be assessed on the side contralateral to the challenge, to serve as a control.

Heat pain threshold and tolerance assessments. A computer-controlled Peltier device (e.g., TSA-II; Medoc, Ramat Yishai, Israel) provides a robust way to measure thermal pain thresholds and tolerance. The probe may be positioned either on the volar aspect of the forearm (site of the capsaicin test) or on the upper thigh (site of the UVB test or the contralateral mirror-image site). The baseline (skin) temperature of the probe is usually held at $32^{\circ} \mathrm{C}$. For threshold measurements, the temperature is gradually increased at a rate of $1{ }^{\circ} \mathrm{C} / \mathrm{s}$ (this rate is chosen to maximize the involvement of nociceptive fibers). ${ }^{22}$ The subject is instructed to respond when the warmth detection and heat pain threshold (first sensation of warmth and heat pain, respectively) or heat pain tolerance (the maximum tolerable pain) are reached. The cutoff temperature for all thermal tests is set to $50^{\circ} \mathrm{C}$, to prevent skin injury. Both the capsaicin test and UVB irradiation have been shown to induce pronounced thermal hyperalgesia, as evidenced by substantially lower heat pain or tolerance thresholds (or both) in the sensitized versus normal skin (see Figure 2 in Chizh et al. ${ }^{12}$ ).

Flare intensity and area. Peripheral nociceptor activation may be assessed by monitoring cutaneous blood flow using imaging techniques such as infrared thermography and laser Doppler imaging (e.g., LDI-2; Moor Instruments, Axminster, Devon, UK). Activation of nociceptive fibers in the skin by capsaicin has been shown to release vasoactive neuropeptides that cause dilatation of small blood vessels in the skin $\left(\right.$ flare $\left.^{23}\right)$. In the case of UVB irradiation, flare is most likely caused by release of prostaglandins ${ }^{24}$; a neurogenic contribution to UVBevoked flare is also possible. ${ }^{25}$ An area extending well beyond the challenge site (i.e., either capsaicin or UVB irradiation) is scanned and the flare area is calculated from all pixels around the stimulation site in which flux values exceeded the 95th percentile of the baseline distribution. The mean blood flow in the area of flare is also calculated using relative flux (arbitrary units). Both the capsaicin challenge test and UVB irradiation induced flare. ${ }^{12}$ In the case of capsaicin, the flare was apparently of axon-reflex type, because the area of flare extended well beyond the area of capsaicin application (see Figure 3 in Chizh et al. ${ }^{12}$ ). With UVB irradiation, the area of flare was largely confined to the site of irradiation.

Sample size considerations. Calculations of the sample size necessary for detection of meaningful changes in test endpoints rely on prior knowledge, which in the case of a novel mechanism may be absent. Indeed, no data had been available for sample size calculations based on effects of TRPV1 antagonists on capsaicinevoked flare. Nonetheless, in the case of heat pain threshold on nonsensitized skin and UVB-induced thermal hyperalgesia, one may use as a benchmark previous data with established analgesics already in clinical use (i.e., opioids and cyclooxygenase inhibitors, respectively). Based on the results from published studies using the UVB model, estimates of between-subject variability for heat pain assessments are between $1.6^{\circ} \mathrm{C}$ and $2.6^{\circ} \mathrm{C}$ and estimates of within-subject variability are between $0.69^{\circ} \mathrm{C}$ and $1.11^{\circ} \mathrm{C}$. Based on these estimates of variability and a sample size of 20 subjects (feasibility-based), it was estimated that the lower and upper bounds of the 95\% confidence interval, for the comparison, SB-705498 versus placebo, would be within $0.7^{\circ} \mathrm{C}$ and $0.9^{\circ} \mathrm{C}$ of the point estimate.

\section{USE OF BIOMARKERS IN SB-705498 EARLY DEVELOPMENT PROGRAM: DID THE PRECLINICAL PHARMACOLOGICAL PROFILE TRANSLATE INTO HUMANS?}

\section{Capsaicin-evoked flare and sensitization}

In the first human study of the TRPV1 antagonist SB-705498, flare evoked by topical dermal administration of capsaicin cream was significantly reduced by SB-705498 versus placebo. $^{12}$ This is consistent with in vitro data on the ability of this compound to competitively antagonize capsaicin-evoked activation of HEK293 cells expressing the human TRPV $1 .{ }^{13}$ The area of flare after SB-7054998 administration was inversely correlated with the plasma levels of the compound, a finding that both confirms that the reduction of flare was due to the active treatment and suggests that the maximum PD efficacy was not achieved.

Notably, the heat hyperalgesia induced by capsaicin was not attenuated by the administration of SB-705498 versus placebo. ${ }^{12}$ This may be explained by a greater 
level of TRPV1 receptor activation by the combined application of heat and capsaicin and by the voltagedependent nature of the SB-705498 antagonism, ${ }^{13}$ which would require higher concentrations of the antagonist to produce detectable effects.

\section{Noxious heat perception}

SB-705498 significantly elevated heat pain thresholds in normal skin when measured at different locations (forearm, thigh), compared with placebo. This effect is consistent with the in vitro data on the blockade by SB-705498 of heat-evoked activation of the TRPV1 receptor. ${ }^{13}$ Unfortunately, no in vivo data on the effect of SB-705498 in preclinical species are available; however, one of the studies of TRPV1 knockout mice demonstrated the receptor involvement in physiological thermonociception. ${ }^{26,27}$ There are also animal data with other TRPV1 selective antagonists demonstrating elevation of the withdrawal reflex threshold in response to noxious heat. ${ }^{28}$ Thus, the alleviation by SB-705498 of heat-evoked pain is in line with the preclinical data and confirms the role of TRPV1 as a heat sensor in humans.

\section{UVB-induced inflammatory hyperalgesia}

SB-705498 significantly increased heat pain threshold and tolerance ( $v s$ placebo) in healthy subjects at the sites that were inflamed after UVB irradiation. ${ }^{12}$ Both heat pain threshold and tolerance were substantially higher in normal skin versus inflamed, a result that implies attenuation of inflammatory hyperalgesia in humans. Again, there was a correlation between this effect and plasma concentration of SB-705498. Because inflammation is known to involve tissue acidification and release of endogenous proinflammatory agents, these results are consistent with the in vitro properties of the TRPV1 receptor, such as its sensitivity to protons $(\mathrm{pH}<6)$ and sensitization by prostaglandins. ${ }^{29-31}$ The in vivo evidence supporting the role of TRPV1 in inflammatory pain comes from studies of TRPV1 knockout mice. ${ }^{26,27}$ As already discussed here, several TRPV1 antagonists have demonstrated reversal of inflammatory hyperalgesia in animal models of inflammatory pain, including SB-705498. ${ }^{14}$ Thus, the effects of SB-705498 in humans confirm the importance of this target in inflammatory pain suggested by the preclinical data.

\section{OTHER TRPV1 ANTAGONISTS}

Although some limited information on PD effects of other TRPV1 antagonists in humans has recently become available, none of the reports mention effects on capsaicin-evoked flare or experimental inflammation. Thus, solid conclusions on the effects of TRPV1 antagonists on those endpoints will require confirmation of the findings on SB-705498 by clinical studies of other compounds of this class. What has been confirmed by brief preliminary reports on other TRPV1 antagonists available so far is the effects on heat pain, and these appear to be consistent with the findings for SB-705498. Thus, AstraZeneca's AZD1386 was investigated in two phase I studies in healthy volunteers and found to increase mean thresholds for warmth detection and heat pain; the effect on heat pain was significant versus placebo (citeline.com, TrialTrove ID-080799 and ID-095917). Importantly, the PD effects of AZD1386 on heat pain persisted after repeated dosing of the compound. Merck-Neurogen have also reported translational PD effects of their TRPV1 antagonist MK-2295 on thermonociception (presentation at Keystone meeting on Neurobiology of Pain and Analgesia, 2009; http://www.keystonesymposia.org). The compound reduced sensitivity to noxious heat in animals (hotplate in rodents and tail immersion in rhesus monkeys) and in healthy human subjects (quantitative thermal sensory tests, pain evoked by hand immersion into or sipping hot water); details of the tests or magnitude of the effects have not yet been reported.

\section{OTHER EXAMPLES: PHASE I TRANSLATIONAL MARKERS OF SODIUM CHANNEL BLOCKADE AND CALCITONIN GENE-RELATED PEPTIDE (CGRP) ANTAGONISM}

Imaging techniques such as infrared thermography and laser Doppler imaging (LDI) can detect minute changes in blood flow (for example, in the skin), related to the release of endogenous vasoactive substances. Because many nociceptive fibers contain vasoactive neuropeptides (e.g., calcitonin gene-related peptide, CGRP), their activation causes axon reflex-mediated vasodilation. ${ }^{32-35}$ Thus, pharmacological agents capable of reducing nociceptor excitability are expected to reduce such axon-reflex vasodilatation. Noxious stimulation of the skin in humans (e.g., using capsaicin or intradermal electrical stimulation) evokes skin flare, which may be attenuated by local or systemic administration of sodium channel blockers and opioids. ${ }^{36-38}$ Importantly, in the case of systemic administration of the sodium channel blocker lidocaine, the effects on flare and on secondary hyperalgesia are observed at clinically efficacious doses of the drug. ${ }^{14,36-39}$ This implies that measures of neurogenic flare and hyperalgesia may serve as predictive PD markers for these and potentially other targets.

One more recent example of using neurogenic skin flare as a translational PD marker is Merck's CGRP antagonist, MK-0974. Given the established role of CGRP in neurogenic inflammation, capsaicin-evoked flare may be used as a marker of CGRP activation in both preclinical species and humans. The compound showed an attenuation of the capsaicin-evoked flare endpoints both in monkeys ${ }^{40}$ and in healthy volunteers in phase I 
study. ${ }^{41}$ The efficacious doses of MK-0974 tested in healthy volunteers were then taken into a clinical study of acute migraine, a condition known to involve neurogenic inflammation of the dura due to a vicious circle of excitation in the trigeminal nociceptive system. ${ }^{42}$ The compound was shown to significantly alleviate headache in migraineurs, with efficacy comparable to that of some triptans ${ }^{42}$ thus confirming the usefulness of translational PD markers for identifying active doses of the CGRP antagonist.

\section{Conclusions}

The use of clinical biomarkers in phase I trials can potentially accelerate the development, or appropriate abandonment, of an analgesic program. The choice of sensory tests and the testing paradigm to show target engagement depends on the preclinical profile of the drug being tested. Nevertheless, PD responses to analgesic therapy may still vary between animal pain models and patients, based in part on species differences and genetic variability within species, differences in PK, differences in pain mechanisms between animal and human models, and differences in environmental influences. These reasons for failure must be distinguished from failure to modulate the target.

The examples discussed in this review confirm the usefulness of clinical biomarkers in the early phase evaluation of novel analgesics for confirmation of target engagement in humans. The ability to assess dose-response and exposure-response relationships has the potential to facilitate the design of dose-finding clinical trials in appropriate pain patients. In some cases, clinical efficacy data exist to support the validity of the dose-selection on the basis of human PD activity. More clinical efficacy data on analgesics with different mechanisms are needed to further confirm the reliability of this approach.

Acknowledgments: This work was funded in part by National Institutes of Health grant NS R01-41503, to C.N.S.

\section{REFERENCES}

1. Kola I, Landis J. Can the pharmaceutical industry reduce attrition rates? Nat Rev Drug Discov 2004;3:711-715.

2. Arendt-Nielsen L, Curatolo M, Drewes A. Human experimental pain models in drug development: translational pain research. Curr Opin Investig Drugs 2007;8:41-53.

3. Arendt-Nielsen L, Yarnitsky D. Experimental and clinical applications of quantitative sensory testing applied to skin, muscles and viscera. J Pain 2009;10:556-572.

4. Huntjens DR, Spalding DJ, Danhof M, Della Pasqua OE. Correlation between in vitro and in vivo concentration-effect relationships of naproxen in rats and healthy volunteers. Br J Pharmacol 2006; 148:396-404.

5. Caterina MJ, Schumacher MA, Tominaga M, Rosen TA, Levine JD, Julius D. The capsaicin receptor: a heat-activated ion channel in the pain pathway. Nature 1997;389:816-824.

6. Szallasi A, Appendino G. Vanilloid receptor TRPV1 antagonists as the next generation of painkillers: are we putting the cart before the horse? J Med Chem 2004;47:2717-2723.
7. Kym PR, Kort ME, Hutchins CW. Analgesic potential of TRPV1 antagonists. Biochem Pharmacol 2009;78:211-216.

8. Gunthorpe MJ, Chizh BA. Clinical development of TRPV1 antagonists: targeting a pivotal point in the pain pathway. Drug Discov Today 2009;14:56-67.

9. Hu HJ, Bhave G, Gereau RW. Prostaglandin and protein kinase A-dependent modulation of vanilloid receptor function by metabotropic glutamate receptor 5: potential mechanism for thermal hyperalgesia. J Neurosci 2002;22:7444-7452.

10. Gunthorpe MJ, Benham CD, Randall A, Davis JB. The diversity in the vanilloid (TRPV) receptor family of ion channels. Trends Pharmacol Sci 2002;23:183-191.

11. Szallasi A, Cruz F, Geppetti P. TRPV1: a therapeutic target for novel analgesic drugs? Trends Mol Med 2006;12:545-554.

12. Chizh BA, O'Donnell MB, Napolitano A, et al. The effects of the TRPV1 antagonist SB-705498 on TRPV1 receptor-mediated activity and inflammatory hyperalgesia in humans. Pain 2007;132: 132-141.

13. Gunthorpe MJ, Hannan SL, Smart D, et al. Characterization of SB-705498, a potent and selective vanilloid receptor-1 (VR1/ TRPV1) antagonist that inhibits the capsaicin-, acid-, and heatmediated activation of the receptor. J Pharmacol Exp Ther 2007; 321:1183-1192.

14. Davis JB, Rami HK, Stevens AJ. SB-705498, a clinical candidate with antagonist activity at TRPV1 and efficacy in a wide range of preclinical pain models. Abstr Soc Neurosci 2005:364.2 (abstract; available at http://www.sfn.org)

15. LaMotte RH, Lundberg LE, Torebjörk HE. Pain, hyperalgesia and activity in nociceptive $\mathrm{C}$ units in humans after intradermal injection of capsaicin. J Physiol 1992;448:749-764.

16. LaMotte RH, Shain CN, Simone DA, Tsai EF. Neurogenic hyperalgesia: psychophysical studies of underlying mechanisms. J Neurophysiol 1991;66:190-211.

17. Simone DA, Baumann TK, LaMotte RH. Dose-dependent pain and mechanical hyperalgesia in humans after intradermal injection of capsaicin. Pain 1989;38:99-107.

18. Sang CN, Gracely RH, Max MB, Bennett GJ. Capsaicin-evoked mechanical allodynia and hyperalgesia cross nerve territories: evidence for a central mechanism. Anesthesiology 1996;85:491-496.

19. Bickel A, Dorfs S, Schmelz M, Forster C, Uhl W, Handwerker HO. Effects of antihyperalgesic drugs on experimentally induced hyperalgesia in man. Pain 1998;76:317-325.

20. Benrath J, Gillardon F, Zimmermann M. Differential time courses of skin blood flow and hyperalgesia in the human sunburn reaction following ultraviolet irradiation of the skin. Eur J Pain 2001;5: $155-167$.

21. Gustorff B, Anzenhofer S, Sycha T, Lehr S, Kress HG. The sunburn pain model: the stability of primary and secondary hyperalgesia over 10 hours in a crossover setting. Anesth Analg 2004;98: 173-177.

22. Yeomans DC, Proudfit HK. Nociceptive responses to high and low rates of noxious cutaneous heating are mediated by different nociceptors in the rat: electrophysiological evidence. Pain 1996;68: 141-150.

23. Geber C, Fondel R, Krämer HH, et al. Psychophysics, flare, and neurosecretory function in human pain models: capsaicin versus electrically evoked pain. J Pain 2007;8:503-514.

24. Hruza LL, Pentland AP. Mechanisms of UV-induced inflammation. J Invest Dermatol 1993;100:35S-41S.

25. Benrath J, Eschenfelder C, Zimmerman M, Gillardon F. Calcitonin gene-related peptide, substance $\mathrm{P}$ and nitric oxide are involved in cutaneous inflammation following ultraviolet irradiation. Eur J Pharmacol 1995;293:87-96.

26. Caterina MJ, Leffler A, Malmberg AB, et al. Impaired nociception and pain sensation in mice lacking the capsaicin receptor. Science 2000;288:306-313.

27. Davis JB, Gray J, Gunthorpe MJ, et al. Vanilloid receptor-1 is essential for inflammatory thermal hyperalgesia. Nature 2000;405: 183-187.

28. García-Martinez C, Humet M, Planells-Cases R, et al. Attenuation of thermal nociception and hyperalgesia by VR1 blockers. Proc Natl Acad Sci U S A 2002;99:2374-2379. 
29. Caterina MJ, Julius D. The vanilloid receptor: a molecular gateway to the pain pathway. Annu Rev Neurosci 2001;24:487-517.

30. Krause JE, Chenard BL, Cortright DN. Transient receptor potential ion channels as targets for the discovery of pain therapeutics. Curr Opin Investig Drugs 2005;6:48-57.

31. Gunthorpe MJ, Szallasi A. Peripheral TRPV1 receptors as targets for drug development: new molecules and mechanisms. Curr Pharm Des 2008;14:32-41.

32. Weidner C, Klede M, Rukwied R, et al. Acute effects of substance $\mathrm{P}$ and calcitonin gene-related peptide in human skin: a microdialysis study. J Invest Dermatol 2000;115:1015-1020.

33. Sauerstein K, Klede M, Hilliges M, Schmelz M. Electrically evoked neuropeptide release and neurogenic inflammation differ between rat and human skin. J Physiol 2000;529:803-810.

34. Schmelz M, Michael K, Weidner C, Schmidt R, Torebjörk HE, Handwerker HO. Which nerve fibers mediate the axon reflex flare in human skin? Neuroreport 2000;11:645-648.

35. Klede M, Clough G, Lischetzki G, Schmelz M. The effect of the nitric oxide synthase inhibitor $N$-nitro-L-arginine-methyl ester on neuropeptide-induced vasodilation and protein extravasation in human skin. J Vasc Res 2003;40:105-114.

36. Koppert W, Dern SK, Sittl R, Albrecht S, Schüttler J, Schmelz M. A new model of electrically evoked pain and hyperalgesia in human skin: the effects of intravenous alfentanil, $S(+)$-ketamine, and lidocaine. Anesthesiology 2001;95:395-402.

37. Koppert W, Ostermeier N, Sittl R, Weidner C, Schmelz M. Lowdose lidocaine reduces secondary hyperalgesia by a central mode of action. Pain 2000;85:217-224.

38. Klede M, Handwerker HO, Schmelz M. Central origin of secondary mechanical hyperalgesia. J Neurophysiol 2003;90:353-359.

39. Challapalli V, Tremont-Lukats IW, McNicol ED, Lau J, Carr DB. Systemic administration of local anesthetic agents to relieve neuropathic pain. Cochrane Database Syst Rev 2005;(4):CD003345.

40. Salvatore CA, Hershey JC, Corcoran HA, et al. Pharmacological characterization of MK-0974 [N-[(3R,6S)-6-(2,3-difluorophenyl)2-oxo-1-(2,2,2-trifluoroethyl)azepan-3-yl]-4-(2-oxo-2,3-dihydro-1 $\mathrm{H}$ imidazo[4,5-b]pyridin-1-yl)piperidine-1-carboxamide], a potent and orally active calcitonin gene-related peptide receptor antagonist for the treatment of migraine. J Pharmacol Exp Ther 2008;324:416-421.

41. de Hoon J, de Lepeleire I, Hickey L, et al. Inhibition of capsaicininduced increase in dermal microvascular blood flow by the oral CGRP antagonist, MK-0974. Cephalalgia 2007;27:738-739 (abstract).

42. Doods H, Arndt K, Rudolf K, Just S. CGRP antagonists: unravelling the role of CGRP in migraine. Trends Pharmacol Sci 2007; 28:580-587. 